\title{
Modal Analysis for TE Volume Modes in Finite-Thickness Slab with Partly Negative Permeability Tensor Component
}

\author{
Masashi Hotta ${ }^{1)^{*}}$, Ryota Ogawa ${ }^{1)}$, Wataru Murai ${ }^{2)}$, and Mitsuo Hano ${ }^{1)}$ \\ 1) Graduate School of Science \& Engineering, Yamaguchi University, Ube 755-8611, JAPAN \\ 2) Toyota Technical Development Co., Toyota 470-0334, JAPAN \\ *hotta@yamaguchi-u.ac.jp
}

\section{Introduction}

Metamaterials composed of periodically accumulated unit-cells have attracted a great deal of attention due to their extraordinary electromagnetic properties [1]-[9]. For example, the most ordinary material has all positive permittivity and permeability, but the material parameters of metamaterial can be negative with properly modifications of unit-cell structure. These metamaterials with negative material parameters can be typically classified into the double-negative material with all negative material parameters and the single-negative material whose either permittivity or permeability is negative.

Excepting waves under the special environment [10], it had been taken for granted that electromagnetic waves can not propagate along the single-negative material, however, it has been presented by the one of authors that the modes with specific polarization can exist on the boundary between the single-negative and double-positive material as surface mode and also on the anisotropic single-negative slab material as volume mode [6]-[9].

In this paper, we have focused on the modal analysis of TE volume modes supported on finite-thickness slab with partly negative and dispersive permeability tensor component. The dispersion characteristics and the dependence for eigenvalues of these volume modes on the slab thickness have been presented numerically and their extraordinary properties would be demonstrated.

\section{Model for Analysis}

\subsection{Basic Structure and Eigenvalue Equation}

As shown in Fig.1, we analyze TE volume modes in slab (region II) with finite thickness $T$, and the tensor permittivity and permeability,

$$
\hat{\varepsilon}=\left(\begin{array}{ccc}
\varepsilon_{x} & 0 & 0 \\
0 & \varepsilon_{y} & 0 \\
0 & 0 & \varepsilon_{z}
\end{array}\right), \quad \hat{\mu}=\left(\begin{array}{ccc}
\mu_{x} & 0 & 0 \\
0 & \mu_{y} & 0 \\
0 & 0 & \mu_{z}
\end{array}\right),
$$

where the coordinate system used for the analysis is also shown in the same figure. It is assumed that both the material tensors are linear and simultaneously diagonalizable and only the components of the permeability tensor are allowed to be negative in region II. The upper and lower regions of the slab (regions I and III) are free-space whose material parameters are $\varepsilon_{0}$ and $\mu_{0}$, respectively.

By solving the Maxwell's equation, the expressions for electromagnetic fields in each region can be obtained. The application of the boundary conditions at $\mathrm{x}=0$ and $\mathrm{x}=-\mathrm{T}$ surfaces for the tangential electromagnetic fields lead us to the following eigenvalue equation for TE 
volume modes,

$$
\left(\mu_{0}^{2} \delta_{1}^{2}-\mu_{z}^{2} \delta_{0}^{2}\right) \sin \delta_{1} T=2 \mu_{0} \mu_{z} \delta_{0} \delta_{1} \cos \delta_{1} T
$$

with $\delta_{0}=\sqrt{\beta^{2}-\omega^{2} \varepsilon_{0} \mu_{0}}$ and $\delta_{1}=\sqrt{\left(\mu_{z} / \mu_{x}\right)\left(\omega^{2} \varepsilon_{y} \mu_{x}-\beta^{2}\right)}$, where $\omega$ is the radial frequency and $\beta$ is the propagation constant, respectively.

\subsection{Material Tensors for the Analysis}

In this paper, since it is assumed that the slab material (region II) is composed of stacked Sprit-Ring-Resonators (SRRs) as shown in Fig.2, only the x-component of the permeability tensor, $\mu_{x}$, would have the dispersion [5], [6] expressed as

$$
\mu_{x}=\mu_{r}(\omega) \mu_{0}=\left\{1-\frac{\pi r^{2}}{a^{2} A(\omega)}\right\} \mu_{0}
$$

with $A(\omega)=\left[1-\left\{3 l c_{0}^{2} / \pi \omega^{2} \ln (2 c / d) r^{3}\right\}\right]$, where $\mathrm{c}_{0}$ is the velocity of light and the other structural parameters are also presented in Fig.2. The resistivity of metallic SRRs is assumed to be zero. In this material, the relative permeability for the x-component of the permeability tensor, $\mu_{r}(\omega)$, shows the negative value over the frequency range from the singular point at $13.48 \mathrm{GHz}$ to $14.41 \mathrm{GHz}$ and is also smaller than -1.0 below $13.92 \mathrm{GHz}$. The other components of the permeability and permittivity tensor are set as $\mu_{y}=\mu_{z}=\mu_{0}$ and $\varepsilon_{x}=1.144 \varepsilon_{0}, \varepsilon_{y}=\varepsilon_{z}=\varepsilon_{0}$, respectively [6].

\section{Modal Properties for TE Volume Modes}

\subsection{Dispersion of Propagation Constants}

By solving the eigenvalue equation (2) numerically under the slab thickness $T=1.0 \mathrm{~mm}$, the dispersion characteristics of TE volume modes are obtained as shown in Fig.3. From this result, as the operating frequency becomes higher, the propagation constants go down toward the cutoff frequency at $13.92 \mathrm{GHz}$. This nature tells us that the obtained TE modes are backward waves.

Figure 4 presents the dispersion characteristics of the TE volume modes with the slab thickness $\mathrm{T}=5.0 \mathrm{~mm}$. From this result, the dispersion curve of $\mathrm{TE}_{1}$ volume mode has a bend, and two eigenvalues can be observed at the same operating frequency. From these facts, the tendency of dispersion curve of $\mathrm{TE}_{1}$ volume mode can be classified into two categories, (i) backward wave behavior and (ii) forward wave behavior.

Figure 5 illustrates the dispersion characteristics of the TE volume modes with slab thickness $\mathrm{T}=10.0 \mathrm{~mm}$ which is much thicker than those of Figs.3 and 4. In this figure, it is much remarkable that the dispersion curve of $\mathrm{TE}_{1}$ mode has disappeared. The other tendencies of the dispersion curves are almost the same as those in Fig.4, excepting the fact that the lowest mode in Fig.5 is the $\mathrm{TE}_{2}$ mode in place of $\mathrm{TE}_{1}$ mode in Fig.4.

\subsection{Dependence on Slab Thickness}

Figure 6 shows the propagation constants of the TE volume modes as a function of the slab thickness, where the operating frequency is kept at $13.60 \mathrm{GHz}$. As illustrated in this figure, there are upper limit of the slab thickness in each mode. Under this situation, as the slab thickness becomes thicker than $5.1 \mathrm{~mm}$, the lowest order $\mathrm{TE}_{1}$ mode turns to show the cutoff operation. From this result, as increasing the slab thickness, the modes disappear from the lower order one. This fact is much different from the property of the volume modes in slab with the double-positive material parameters. 


\section{Conclusions}

The modal properties of TE volume mode propagating along a Single-Negative slab with negative and dispersive component of permeability tensor were demonstrated in this paper.

By investigating the dispersion characteristics of the eigenvalues, it has been brought to light that most of the TE modes in this structure have backward wave nature, but some waves in thick slab are the forward wave. Furthermore, by inspecting the modal dependence of this structure as a function of slab thickness, the modes sequentially disappear from the lower order one as the slab thickness becomes thicker.

These properties are much similar to those of Magnetostatic Wave (MSW) in the magnetic substance. So it seems that presented metamaterial composed of the nonmagnetic unit cells works as the magnetic substance.

\section{Acknowledgement}

This work was partly supported by the Ministry of Education, Culture, Sports, Science, and Technology (MEXT), Japan, Grant-in-Aid for Scientific Research (C) 18560335.

\section{References}

[1] V.G. Veselago, "The electrodynamics of substances with simultaneously negative values of $\varepsilon$ and $\mu$," Soviet Physics Uspekhi, vol.10, no.4, pp.509-514, 1968.

[2] A. Alu and N. Engheta, "Guided modes in a waveguide filled with a pair of single-negative (SNG), double-negative (DNG), and/or double-positive (DPS) layers," IEEE Trans. Microwave Theory Tech., vol.52, no.1, pp.199-210, Jan.2004.

[3] C. Caloz and T. Itoh, Electromagnetic Metamaterials: Transmission Line Theory and Microwave Applications, John Wiley \& Sons, 2006

[4] R. A. Shelby, D. R. Smith, and S. Schults, "Experimental verification of a negative index of refraction," Science, vol.292, pp.77-79, 2001.

[5] J. B. Pendry, A. J. Holden, D. J. Robbins, and W. J. Stewart, "Magnetism from conductors and enhanced nonlinear phenomena," IEEE Trans. Microwave Theory Tech., vol.47, no.11, pp.2075-2081, Nov.1999.

[6] M. Hotta, M. Hano, and I. Awai, "Modal Analysis of Finite-Thickness Slab with Single-Negative Tensor Material Parameters," IEICE Trans. Electron., vol.E89-C, no.9, pp.275-278, Sept.2006.

[7] M. Hotta, M. Hano, and I. Awai, "Modes in Single-Negative Slab with Tensor Material Parameters," Proc. 2006 European Microwave Conf. (Manchester, UK), no.EuMC54-1, pp.1007-1010, Sept. 2006.

[8] M. Hotta, M. Hano, and I. Awai, "Surface Waves along a Boundary of Single-Negative and Double-Positive Materials," Proc. 2004 European Microwave Conf. (Amsterdam, Netherlands), no.EuMC05-01, pp.439-442, Oct. 2004.

[9] M. Hotta, M. Hano, and I. Awai, "Surface waves along a boundary of single negative material," IEICE Trans. Electron., vol.E88-C, no.2, pp.275-278, Feb. 2005.

[10] C. Kittel, Introduction to Solid State Physics, $7^{\text {th }}$ ed., John Wiley \& Sons, 1995. 


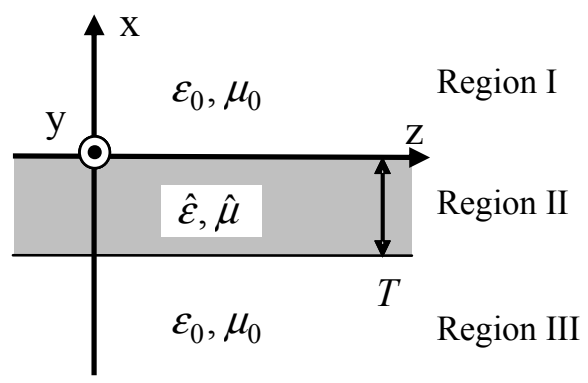

Fig.1 Finite-Thickness slab with tensor material parameters.
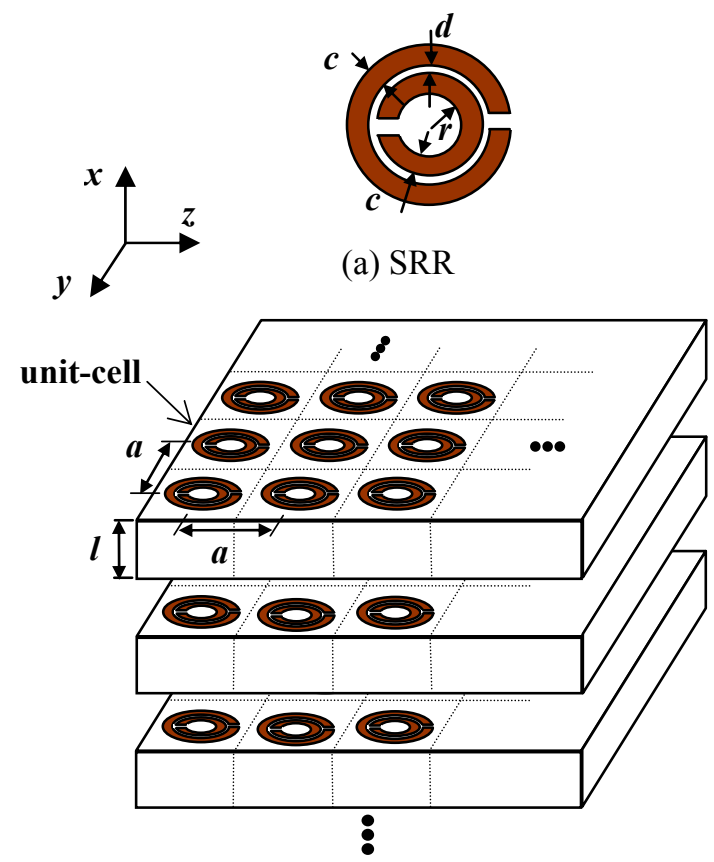

$a=1.0 \times 10^{-3} \mathrm{~m}, c=1.0 \times 10^{-4} \mathrm{~m}, d=1.0 \times 10^{-5} \mathrm{~m}$ $l=2.0 \times 10^{-6} \mathrm{~m}, r=2.0 \times 10^{-4} \mathrm{~m}$

(b) Stacking and arranging sequence

Fig.2 Structure of metamaterial with negative permeability with SRR array.

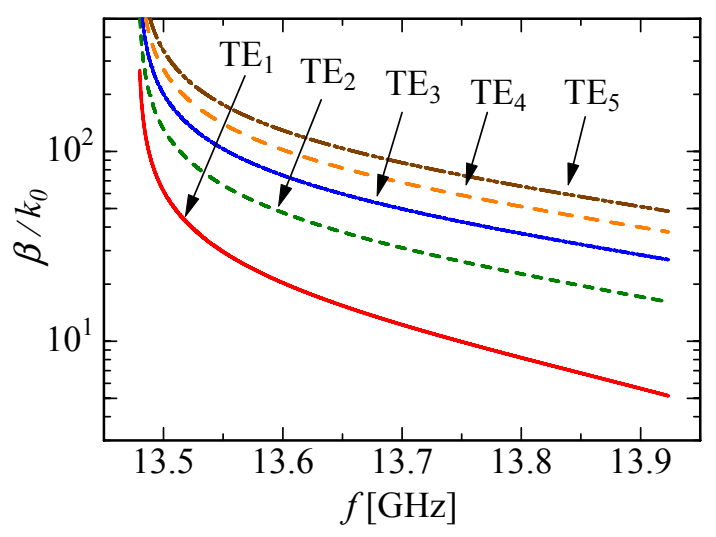

Fig.3 Dispersion characteristics of the TE volume modes with the slab thickness $\mathrm{T}=1.0 \mathrm{~mm}$.

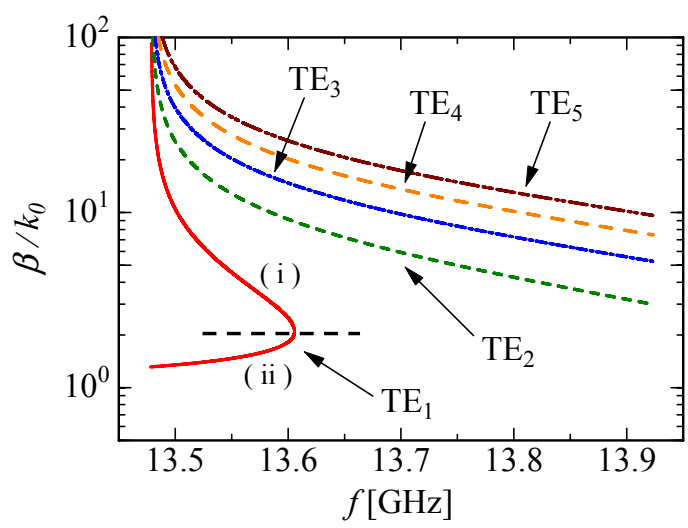

Fig.4 Dispersion characteristics of the TE volume modes with the slab thickness $\mathrm{T}=5.0 \mathrm{~mm}$.

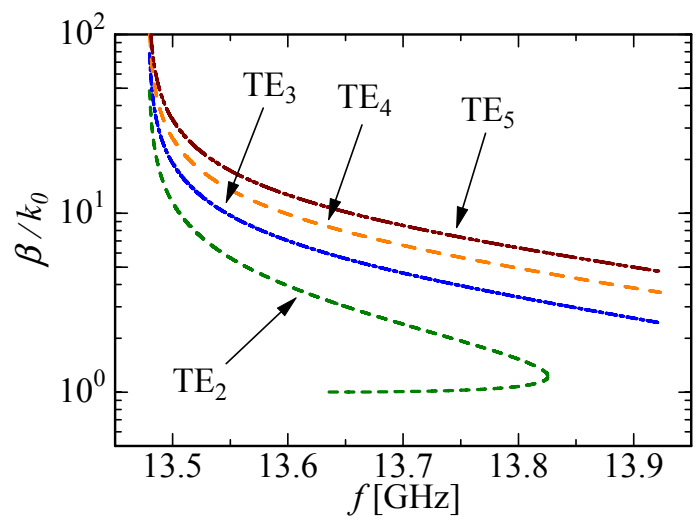

Fig.5 Dispersion characteristics of the TE volume modes with the slab thickness $\mathrm{T}=10.0 \mathrm{~mm}$.

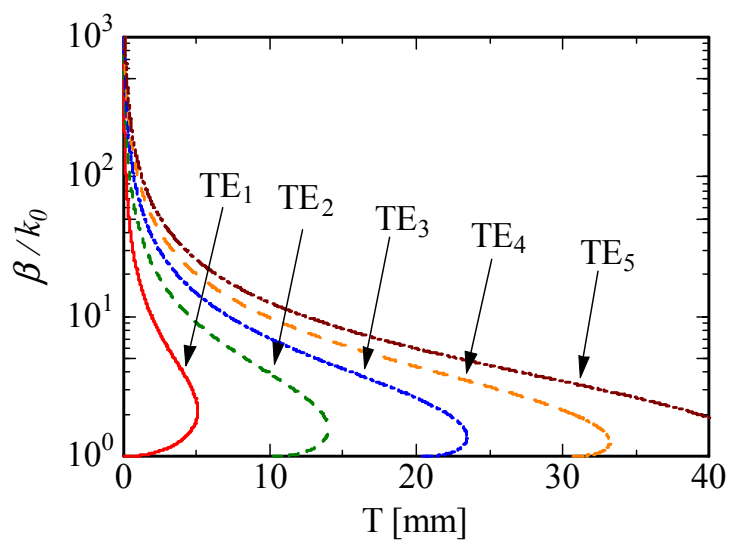

Fig.6 Modal dependence of Anisotropic single-negative slab on its thickness at $\mathrm{f}=13.60 \mathrm{GHz}$. 